\title{
Prevalência de Sobrepeso e Obesidade em Pré-Escolares do Município de Frei Paulo-SE: Um Estudo Longitudinal
}

\author{
Prevalence of Overweight and Obesity Among Preschool Children in the \\ Municipality of Frei Paulo, SE: A Longitudinal Study
}

\author{
ISABELLA FONTES DE SANTANA LINS ${ }^{1}$ \\ LAÍS LIMA DE OLIVEIRA² \\ GÊNITON SANTOS LIMA ${ }^{3}$ \\ TAUANNY ARAGÃO DE MOURA ${ }^{4}$ \\ DIEGO MOURATANAJURA ${ }^{5}$
}

\section{RESUMO}

Objetivos: Avaliar o perfil antropométrico de pré-escolares de quatro a seis anos da Escola Municipal Juvina Moreira, em Frei Paulo-SE. Materiais e métodos: Trata-se de um estudo longitudinal que acompanhou 42 crianças durante seis meses, no qual foram realizadas duas coletas de dados antropométricos. Os dados de peso e altura foram avaliados segundo os percentis propostos pela Organização Mundial da Saúde para o Índice de Massa Corporal. Os pontos de corte utilizados foram: percentil menor que três para déficit de peso; percentil entre três e 85 para eutróficos; e percentil e" 85 para risco ou excesso de peso. Para análise estatística foi utilizado o programa Graph Pad Prism V.5.0. O teste de Mann Whitney foi usado para comparação entre duas variáveis e o coeficiente de Spearman para correlação entre o índice CA/altura e o escore-Z do IMC. O nível de significância adotado foi de $5 \%$. Resultados: Das 42 crianças que participaram do estudo, $18(42,86 \%)$ foram do sexo feminino e $24(57,14 \%)$ do sexo masculino. No primeiro semestre, $26,19 \%$ dos pré-escolares foram classificados com risco de sobrepeso ou excesso de peso (sobrepeso ou obesidade). No segundo semestre, esse número caiu para $16,67 \%$. A prevalência de circunferência alterada se manteve em 19\% nos dois semestres, e foi encontrada uma correlação positiva entre a relação circunferência abdominal por altura e o escore-z do Índice de Massa Corporal. Conclusão: Percebeuse que as taxas de sobrepeso e obesidade nos pré-escolares de Frei Paulo são relevantes e compatíveis com o encontrado em outras regiões do Brasil. Apesar da redução dessas taxas no segundo semestre, os números evidenciam a necessidade de intervenção nessa população através da educação e promoção da saúde.

DESCRITORES

Obesidade.Sobrepeso.Criança.Saúde Pública.

\begin{abstract}
Objective: To evaluate the anthropometric profile of preschool children aged four to six years at Juvina Moreira Municipal School, in the city of Frei Paulo, SE, Brazil. Material and Methods: This was a longitudinal study that followed up 42 children for six months, in which two anthropometric data collections were performed. The data obtained were analyzed based on cut-off points established by the World Health Organization for Body Mass Index. The cut-off points used were: percentile lower than three for weight deficit; percentile between three and 85 for eutrophic; and percentile e"85 for risk or development of overweight. Mann-Whitney test was used to compare two variables and the Spearman coefficient was used to test a correlation between waist: height ration and the BMI Z-score. The data were analyzed using GraphPad Prism version 5.0, with a 5\% significance level. Results: Out of the 42 children who participated in the study, 18 (42.86\%) were females and 24 (57.14\%) were males. In the first semester, $26.19 \%$ of the pre-school children were classified as at risk of overweight or out of weight range (overweight or obesity). In the second semester, this number decreased to $16.67 \%$. The prevalence of altered circumference remained at $19 \%$ in both semesters, and a positive correlation was found between the waist circumference ratio and the BMI Z-score. Conclusion: The overweight and obesity rates observed among pre-school children from Frei Paulo are relevant and compatible to what is found in other regions of Brazil. Despite the reduction of these rates in the second semester, the numbers show the need for intervention in this population through education and health promotion.
\end{abstract}

\section{DESCRIPTORS}

Obesity.Overweight.Child.Public Health.

1 Graduanda em Medicina.Universidade Federal de Sergipe-UFS. Lagarto.Sergipe.Brasil.

2 Graduanda em Enfermagem. Universidade Federal de Sergipe-UFS. Lagarto.Sergipe.Brasil.

3 Agente Comunitário de Saúde. Secretaria Municipal de Saúde. Frei Paulo.Sergipe.Brasil.

4 Médica. Residente em Clínica Médica pela Universidade Federal de São Paulo-UNIFESP.São Paulo.São Paulo. Brasil.

5 Professor do Departamento de Educação em Saúde da Universidade Federal de Sergipe -UFS.Lagarto.Sergipe. Brasil. 
A obesidade é uma doença crônica nãotransmissível caracterizada pelo acúmulo excessivo de gordura capaz de representar sérios riscos à saúde e a qualidade de vida do indivíduo ${ }^{1,2}$. Sua prevalência vem crescendo em todo o mundo, tanto em países desenvolvidos como em desenvolvimento, sendo por isso considerada problema de saúde pública ${ }^{3}$.

No Brasil o cenário é o mesmo, o número de obesos tem aumentado em todas as regiões do país. Segundo pesquisa realizada pela Vigilância de Fatores de Risco e Proteção para Doenças Crônicas por Inquérito Telefônico (Vigitel)4, em 2016, mais da metade da população brasileira com mais de 18 anos estava acima do peso (53,8\%). O mesmo estudo revelou, ainda, uma prevalência de obesidade de 18,9\% para a mesma faixa etária. Esse quadro, entretanto, não se restringe apenas à faixa etária adulta, e as taxas de obesidade na infância e adolescência sofreram notório aumento nos últimos anos ${ }^{6}$. No último levantamento realizado pelo Instituto Brasileiro de Geografia e Estatística (IBGE) $)^{5}$, entre $2008 / 2009,32 \%$ das crianças de cinco a nove anos apresentavam excesso de peso e $11,8 \%$ eram obesas. Na faixa etária de 10 a 19 anos, esse número foi de $20,5 \%$ para excesso de peso e de $4,9 \%$ para obesidade. Quanto ao panorama da obesidade infantil na cidade de Frei Paulo, um estudo transversal realizado com 975 escolares e pré-escolares de 4 a 17 anos demonstrou que $22,15 \%$ estavam acima do peso e $5,23 \%$ estavam abaixo do peso ideal para a idade ${ }^{7}$.

O aumento da obesidade infanto-juvenil é um fato preocupante, uma vez que são grandes as chances dessa criança ou adolescente se tornar um adulto obeso ${ }^{8,9}$. Um estudo finlandês publicado em 2014 constatou que pré-escolares de 3 a 5 anos que apresentam um Índice de Massa Corporal (IMC) acima do percentil 90 possuem um maior risco de desenvolver obesidade central na vida adulta, bem como síndrome metabólica. O estudo demonstrou também que cerca de um terço das crianças que estão acima do percentil 95 aos cinco anos será obeso aos 31 anos e cerca de um quarto terá síndrome metábólica nesta idade ${ }^{10}$.

Vários fatores podem estar relacionados ao desenvolvimento da obesidade no indivíduo. Dentre eles, podemos destacar a herança genética, hábitos alimentares inadequados e falta de exercício físico ${ }^{11}$. Quanto ao diagnóstico, é necessário identificar os níveis de risco da doença, tendo para isso diversos métodos de quantificação. O IMC é um índice simples, prático e barato, bastante utilizado em estudos que visam avaliar o estado nutricional em pré-escolares, sendo, portanto, bem aceito na comunidade científica ${ }^{12,13}$. Entretanto, apesar de ser o método mais utilizado para análise da composição corporal para fins epidemiológicos, sua interpretação, quando individual, precisa ser feita com cautela, uma vez que trata-se de uma manipulação matemática que pressupõe que todo excedente de peso corporal no indivíduo consiste no aumento de gordura ${ }^{14}$. A medida de circunferência abdominal reflete melhor o conteúdo de gordura visceral, e pode ser utilizada em associação com o IMC, visando reduzir as limitações de cada uma das avaliações quando utilizadas isoladamente ${ }^{11}$.

Levando em conta o panorama atual da obesidade e as inúmeras complicações desenvolvidas por essa doença crônica nãotransmissível, percebe-se a necessidade de uma intervenção mais direta na sociedade. A prevenção, o diagnóstico e o tratamento precoces da obesidade se tornam fundamentais ${ }^{15}$. Diante do que foi exposto, o objetivo desse estudo é conhecer o estado nutricional dos pré-escolares de uma escola municipal do interior de Sergipe.

\section{MATERIAIS E MÉTODOS}

Este estudo faz parte do projeto "Saúde nas escolas: combatendo o excesso de peso", que visa avaliar os fatores condicionantes do excesso de peso infanto-juvenil na cidade de Frei Paulo-SE, assim como promover palestras e grupos de discussões alertando a população sobre os riscos do sobrepeso/obesidade e suas complicações.

Estudo longitudinal realizado na Escola Municipal Juvina Moreira, único pré-escolar público da região urbana da cidade de Frei Paulo. Foram realizadas duas avaliações, com intervalo de seis meses, nas quais foram coletadas medidas antropométricas (peso, altura e circunferência abdominal) de pré-escolares de quatro a seis anos matriculados no Alfa I no ano de 2015. Dos 77 alunos matriculados nesta série, foram incluídos aqueles que estavam presentes nos dois dias de coleta e cujos pais ou responsáveis legais assinaram o Termo de Consentimento Livre e Esclarecido. $O$ estudo foi aprovado pelo comitê de ética em Pesquisa do Hospital Universitário de Aracaju/Universidade Federal de Sergipe (CAEE ${ }^{\circ}$ 37644114.2.0000.5546). A amostra inicial foi composta de 53 crianças, tendo uma perda de $20,75 \%$ no segundo semestre. Desta forma, o presente estudo foi composto pelos 42 pré- 
escolares que participaram das duas avaliações (maio/novembro).

Durante a avaliação clínica, foi utilizada uma balança digital da marca Multilaser com precisão de $100 \mathrm{~g}$ para obter a massa corporal das crianças (em kg), na qual elas subiram descalças, mantendo os pés justapostos e vestindo roupas leves; para a altura $(\mathrm{em} \mathrm{cm})$, foi utilizado um estadiômetro de parede da marca Wiso com precisão de $1 \mathrm{~mm}$. A circunferência abdominal (CA) $(\mathrm{em} \mathrm{cm})$ foi mensurada com uma fita métrica não elástica da marca Premium. Pediu-se para a criança ficar em posição supina e mediu-se o ponto médio entre o rebordo costal inferior e a crista ilíaca, ao final da expiração.

Os dados de peso e altura foram avaliados segundo os percentis propostos pela Organização Mundial da Saúde (OMS) para o IMC. Os pontos de corte utilizados foram: percentil menor que três para déficit de peso; percentil ente três e 85 para eutróficos; e percentil e" 85 para risco ou excesso de peso ${ }^{16,17}$. Para a avaliação da CA, foi utilizado os pontos de corte propostos por Taylor et al. ${ }^{18}$. Crianças acima do percentil 80, de acordo com a idade e o sexo, foram classificadas com valor de CA elevado. Foi calculada ainda a relação CA/altura, índice que ganhou notoriedade, uma vez que permite identificar uma maior predisposição para problemas cardiometabólicos $^{19}$. Para isso, foi utilizado o ponto de corte universal proposto por Ashwell et al. ${ }^{20}$, que corresponde a 0,5 para ambos os sexos.

Para análise estatística, foi utilizado o programa GraphPad Prism V.5.0. O teste de Mann Whitney foi utilizado para avaliar as diferenças entre dois grupos. Para avaliação da correlação entre o índice CA/altura e o escore-z do IMC utilizou-se o coeficiente de correlação de Spearman. Um valor de $p<0,05$ foi considerado significativo. $\mathrm{Na}$ avaliação do estado nutricional, foi utilizado o software AnthroPlus (para escolares de cinco a seis anos) e Anthro (para escolares de quatro anos) (WHO AnthroPlus/Anthro for personal computers: software for assessing growth of the world's children and adolescentes. Genebra, Suíça) e os pontos de corte definidos para IMC/idade pela OMS ${ }^{21}$.

\section{RESULTADOS}

Das 42 crianças avaliadas no estudo, 18 $(42,86 \%)$ eram do sexo feminino e $24(57,14 \%)$ do sexo masculino. A média de peso, altura e IMC foram estatisticamente semelhantes para ambos os sexos. Quanto ao estado nutricional, apenas uma criança estava abaixo do peso $(2,38 \%)$ no primeiro semestre de 2015 , enquanto $26,19 \%$ dos pré-escolares foram classificados com risco de sobrepeso ou excesso de peso (Figura 1). Destes, três $(7,14 \%)$ eram obesos e quatro $(9,52 \%)$ apresentavam sobrepeso.

No segundo semestre, o número de préescolares com risco de sobrepeso ou excesso de peso caiu para 16,67\% (Figura 1). Das crianças que estavam acima do peso, apenas uma era obesa $2,38 \%$ e três $(7,14 \%)$ foram classificadas com sobrepeso. Em relação à magreza, houve mudança quanto à distribuição por sexo. Enquanto apenas uma criança do sexo feminino estava abaixo do peso no primeiro semestre, um menino e nenhuma menina apresentava magreza no segundo semestre.

A frequência de sobrepeso e obesidade foi maior no sexo feminino nos dois semestres. Inicialmente foi encontrado que $11,11 \%$ das meninas apresentavam sobrepeso e $11,11 \%$ eram obesas. Nessa mesma época, a taxa de sobrepeso e obesidade no sexo masculino foi de $8,33 \%$ e $4,17 \%$, respectivamente. No segundo semestre, $11,11 \%$ das meninas e $4,17 \%$ dos meninos estavam com sobrepeso; a taxa de obesidade foi de 5,56\% para elas e $4,17 \%$ para eles (Tabela 1 ).

Quanto aos valores de CA, 19\% das crianças apresentaram medida de circunferência abdominal alterada, tanto no primeiro quanto no segundo semestre de acordo com os pontos de corte propostos por Taylor et al. ${ }^{16}$. Ao utilizar o índice CA/altura, $33,3 \%$ dos pré-escolares possuíam valor maior ou igual a 0,5 , o que configura risco aumentado para doenças cardiovasculares. Esse valor, no segundo semestre, caiu para $16,67 \%$.

Percebeu-se uma diferença significativa entre as CAs médias do grupo eutrófico ou baixo peso $(73,80 \%)$ e do grupo com risco ou excesso de peso $(26,19 \%)$ no primeiro semestre, com $p<0,0001$ (Figura 2). Foi encontrada também uma correlação positiva entre a razão CA/A e o escore$z$ do IMC, indicando que os alunos com os maiores valores de CA/A possuíam os maiores valores de $z$-score do IMC ( $r$ de Spearman: 0,73, $p<0,0001$ ) (Figura $3 \mathrm{~A}$ ). No segundo semestre, encontrou-se novamente uma diferença significativa entre as $\mathrm{CAs}$ médias do grupo eutrófico ou magro $(83,33 \%)$ e do grupo com risco ou excesso de peso (16,67\%), com $p<0,0001$ (Figura 2). Foi encontrada também uma correlação positiva entre a razão CA/A e o escore$z$ do IMC ( $r$ de Spearman: 0,70, $p<0,0001$ ) (Figura $3 \mathrm{~B})$. 


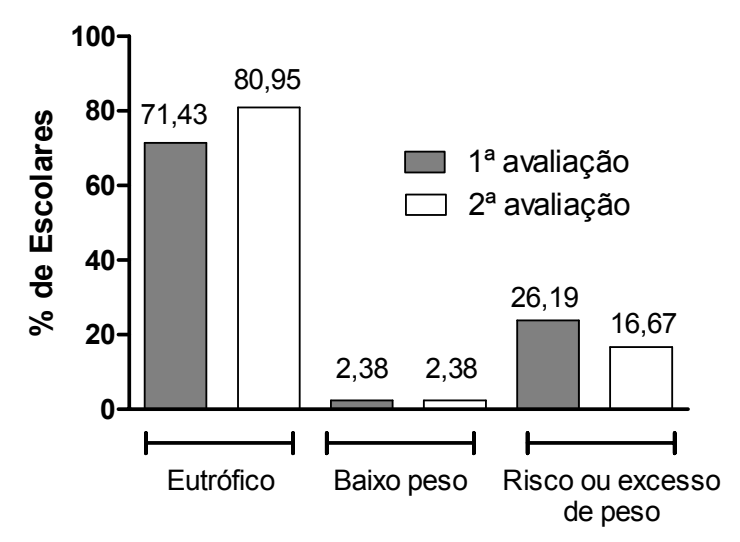

Figura 1. Distribuição do estado nutricional de préescolares da Escola Municipal Professora Juvina Moreira, no primeiro ( $1^{a}$ avaliação) e segundo ( $2^{a}$ avaliação) semestre de 2015.

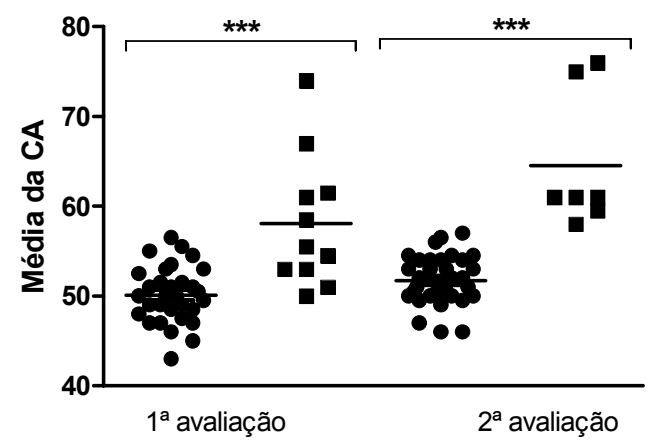

Figura 2. Comparação das circunferências abdominais (CA) entre as crianças classificadas sem excesso de peso e as com risco ou excesso de peso, no primeiro ( $1^{a}$ avaliação) e segundo $\left(2^{a}\right.$

- Sem excesso de peso avaliação) semestre de 2015. $\left.{ }^{* * *}, p<0,0001\right)$.

- Com risco ou excesso de peso



$1^{\mathrm{a}}$ avaliação
B)

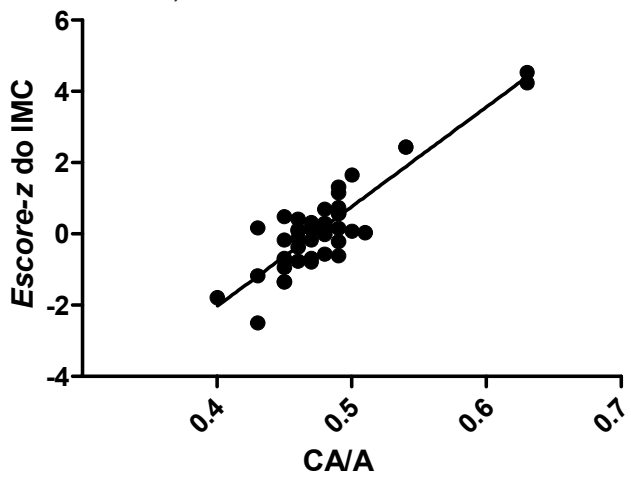

$2^{\mathrm{a}}$ avaliação

Figura 3. Correlação entre CA/A e escore-z dos pré-escolares. (A) No primeiro semestre de 2015 ( $r$ de Spearman: 0,73, $p<0,0001)$. (B) no segundo semestre de 2015 ( $r$ de Spearman: 0,70, $p<0,0001$ ). 


\begin{tabular}{|c|c|c|c|c|c|c|c|c|c|c|c|c|}
\hline \multirow{3}{*}{ Classificação } & \multicolumn{6}{|c|}{$1^{\circ}$ Semestre } & \multicolumn{6}{|c|}{$2^{\circ}$ Semestre } \\
\hline & \multicolumn{2}{|c|}{$\begin{array}{c}\text { Meninas } \\
\text { (18) }\end{array}$} & \multicolumn{2}{|c|}{$\begin{array}{l}\text { Meninos } \\
\text { (24) }\end{array}$} & \multicolumn{2}{|c|}{ Total (42) } & \multicolumn{2}{|c|}{$\begin{array}{c}\text { Meninas } \\
\text { (18) }\end{array}$} & \multicolumn{2}{|c|}{$\begin{array}{l}\text { Meninos } \\
\text { (24) }\end{array}$} & \multicolumn{2}{|c|}{ Total (42) } \\
\hline & $n$ & $\%$ & $n$ & $\%$ & $n$ & $\%$ & $\mathbf{n}$ & $\%$ & $\mathbf{n}$ & $\%$ & $\mathbf{n}$ & $\%$ \\
\hline Magreza & 1 & 5,56 & 0 & 0 & 1 & 2,38 & 0 & 0 & 1 & 4,17 & 1 & 2,38 \\
\hline Eutrofia & 11 & 61,11 & 19 & 79,17 & 30 & 71,43 & 14 & 77,78 & 20 & 83,33 & 34 & 80,95 \\
\hline $\begin{array}{l}\text { Risco de } \\
\text { sobrepeso }\end{array}$ & 2 & 11,11 & 2 & 8,33 & 4 & 9,52 & 1 & 5,56 & 1 & 4,17 & 2 & 4,76 \\
\hline Sobrepeso & 2 & 11,11 & 2 & 8,33 & 4 & 9,52 & 2 & 11,11 & 1 & 4,17 & 3 & 7,14 \\
\hline Obesidade & 2 & 11,11 & 1 & 4,17 & 3 & 7,14 & 1 & 5,56 & 1 & 4,17 & 2 & 4,76 \\
\hline
\end{tabular}

\section{DISCUSSÃO}

O presente estudo avaliou a prevalência de risco, sobrepeso e obesidade em pré-escolares de uma escola municipal de Frei Paulo-SE no primeiro e segundo semestre de 2015. Essa faixa etária é de extrema relevância, uma vez que é nesse período que as crianças desenvolvem seus hábitos alimentares e sofrem bastante influência do meio em que vivem ${ }^{22}$.

A literatura é escassa quanto a estudos longitudinais sobre obesidade e sobrepeso nessa faixa etária, a maioria deles consistem em estudos transversais, que avaliam a frequência de sobrepeso e obesidade em pequenos grupos populacionais ${ }^{6,7,23,25,27}$. Um dos poucos trabalhos do tipo longitudinal sobre obesidade e sobrepeso em crianças foi publicado recentemente por Cunningham et al. ${ }^{24}$, no qual 7.738 crianças americanas matriculadas na pré-escola foram acompanhadas por nove anos. Como conclusão do estudo, os pesquisadores afirmaram que crianças com excesso de peso em idade pré-escolar têm quatro vezes mais risco de se tornarem obesos por volta dos 14 anos. Esse resultado contrasta com o encontrado em Frei Paulo, onde houve redução da prevalência seis meses após a primeira avaliação.
Esse fato pode estar relacionado ao pequeno tamanho amostral do presente estudo. Outro ponto a ser considerado é o aumento da estatura dessas crianças, compatível com a fase do desenvolvimento, o qual prevaleceu sobre o ganho de peso durante o curto intervalo de seis meses. Além disso, os projetos educativos desenvolvidos pelo grupo de pesquisa (palestras educativas sobre alimentação saudável e a importância da prática de atividade física) também podem ter contribuído para a redução do IMC dessas crianças.

A porcentagem de pré-escolares com risco de sobrepeso ou excesso de peso encontrada no primeiro semestre em Frei Paulo foi de $26,19 \%$, valor próximo ao encontrado em um estudo com pré-escolares da região semiárida do Nordeste ${ }^{25}$, cuja prevalência foi de $28,5 \%$. No entanto, ao contrário do encontrado em Frei Paulo, a maioria dos alunos estavam com risco de sobrepeso $(19,9 \%)$, em vez de excesso de peso $(8,6 \%)$.

No segundo semestre, a ocorrência de crianças com excesso de peso sofreu uma redução, caindo de $16,67 \%$ (7,14\% obesas e $9,52 \%$ com sobrepeso) para $11,90 \%$ (4,76\% obesas e $7,14 \%$ com sobrepeso). O mesmo foi observado em outro estudo que acompanhou pré-escolares durante três anos, no qual a porcentagem de obesos passou de 
$6,4 \%$ para $4,5 \%{ }^{26}$. Quanto à estratificação por sexo, o número de crianças obesas em Frei Paulo foi maior no sexo feminino, semelhante ao encontrado no estudo de $\mathrm{Cunha}^{27}$ que contou com 41 préescolares de dois a cinco anos de idade. Nele, observou-se uma prevalência da obesidade de $40 \%$ para meninas e de $30,42 \%$ para meninos.

No que diz respeito à desnutrição, os números variam de acordo com as regiões do país. Aquelas regiões mais desenvolvidas como o Sul e Sudeste apresentam as menores taxas de magreza e magreza extrema, variando de 2 a $3 \%$; no Nordeste, por sua vez, o percentual de indivíduos abaixo do peso chega a cerca de $5 \%{ }^{5}$. Em Frei Paulo, esse número foi de $2,38 \%$ nos dois semestres de 2015, sendo, portanto, compatível com o Sul e Sudeste. O tamanho amostral reduzido pode explicar novamente os resultados encontrados, uma vez que em trabalho prévio desenvolvido com crianças de 4 a 17 anos da cidade de Frei Paulo, obteve-se valores similares ao observado na região Nordeste ${ }^{7}$.

Estudos que visam avaliar a circunferência abdominal de pré-escolares são escassos na literatura; além disso, não existe uma padronização internacional de pontos de corte para classificação de adiposidade abdominal em crianças $^{28}$. Trabalho realizado com 1.408 préescolares e escolares demonstrou que 13,3\% apresentavam valor de CA alterado, tendo como ponto de corte o percentil $90^{29}$. O presente estudo utilizou o percentil 80 , apresentando desta forma uma maior pevalência de CA alterada (19\%). Entretanto, ao utilizar o percentil 90 nos préescolares de Frei Paulo, observou-se que apenas $11,9 \%$ estavam com CA alterada, valor semelhante ao encontrado no trabalho acima citado. Dias et al. ${ }^{30}$ estudou a circunferência de cintura (medida obtida na altura da cicatriz umbilical) de pré- escolares de Botucatu-SP, obtendo uma correlação forte entre IMC e adiposidade abdominal, semelhante ao encontrado em Frei Paulo, onde as crianças com excesso de peso tinham os maiores valores de CA. As medidas de CA/A também estavam fortemente correlacionadas com o IMC, equivalente ao que foi encontrado no estudo de Santomauro et al. ${ }^{19}$

O presente estudo apresentou algumas limitações. A primeira delas foi o tamanho da amostra, uma vez que houve perdas de participantes ao longo do trabalho. Além disso, apesar da cidade ter poucos habitantes e esta ser a única escola pré-escolar pública da zona urbana, o estudo poderia ter sido ampliado para avaliar crianças de escolas privadas ou públicas descentralizadas. Faltou também conhecer mais sobre o perfil de cada estudante e acompanhá-lo por um período maior de tempo. A aplicação de questionários com os pais dos alunos forneceria informações importantes sobre dieta e hábitos de vida, podendo relacioná-las com o estado nutricional das crianças.

\section{CONCLUSÃO}

Pode-se concluir que a prevalência de sobrepeso e obesidade nos pré-escolares da cidade de Frei Paulo é maior que a prevalência de magreza, apesar do baixo peso para idade ainda estar presente e representar um grave problema de saúde pública. A redução das taxas de sobrepeso e obesidade é um ponto relevante, uma vez que foi de encontro aos resultados obtidos em outros estudos. Entretanto, é importante ressaltar que esses números ainda merecem atenção por parte dos programas locais de saúde e evidenciam a necessidade de atividades de intervenção nessa população, através da educação e promoção da saúde. 


\section{REFERÊNCIAS}

1. WHO. Obesity [Internet]. Health Topics. 2008. Michels N, Susi K, Marques-Vidal PM, Nydegger A, Puder JJ. Psychosocial Quality-of-Life, Lifestyle and Adiposity: A Longitudinal Study in Pre-schoolers (Ballabeina Study). Int J Behav Med. 2016;23(3):383-92.

2. Niehues JR, Gonzales AI, Lemos RR, Bezerra PP, Haas P. Prevalence of overweight and obesity in children and adolescents from the age range of 2 to 19 years old in Brazil. Int J Pediatr [Internet]. 2014.

3. VIGITEL Brasil. Vigilância de Fatores de Risco e Proteção para Doenças Crônicas por Inquerito Telefônico. Ministério da Saúde. 2017. 162 p.

4. IBGE. Pesquisa de Orçamentos Familiares 2008-2009. Ministério da Saúde. 2009. 130p.

5. Malinski MPVR. Sobrepeso e obesidade em jovens escolares. Arq Ciência da Saúde. 2016;23(1):68-72.

6. Ribeiro BVS, Mendonça RG, Oliveira LL. Anthropometry and lifestyle of children and adolescent in inland of Northeastern Brazil. J Hum Growth Dev. 2017;27(1): Ahead of print.

7. Matoso LML, Oliveira LEDC, Bezerra CMV. O enfermeiro nas ações da saúde do indivíduo, família e comunidade: Obesidade exógena infanto-juvenil e seus hábitos alimentares. Rev Científica da Esc da Saúde. 2014;1(1):67-80.

8. Damiani D. Obesidade na Infância e Adolescência - Um Extraordinário Desafio! Arq Bras Endocrinol Metab. 2000;44(5):363-5.

9. Gravercer L, Sørensen TIA, Petersen L, Sovio U, Kaakinen M, Sandbaek A, et al. Preschool Wheight and Body Index in Relation to Central Obesity and Metabolic Syndrom in Adulthood. 2014; 9(3).

10. Langley-Evans SC, Moran VH. Childhood obesity: risk factors, prevention and management. J Hum Nutr Diet [Internet]. 2014 Oct;27(5):411-2.

11. Telles RK, Barros Filho A de A. O uso da antropometria como método de avaliação da composição corporal em Pediatria. Revista de Ciências Médicas. 2003;12(4):351363.

12. Andaki ACR, Tinoco ALA, Mendes EL, Priore SE,
Franceschini S do CC, Santana LF da RS. Metodologias para avaliação da composição corporal em crianças. Revista Digital. 2011;156(16).

13. Sant'anna M de SL, Priore SE, Franceschini $S$ do CC. Métodos de avaliação da composição corporal em crianças. Rev. Paul. Pediatr. [Internet]. 2009;27(3):315321.

14. Güngör NK. Overweight and obesity in children and adolescents. J Clin Res Pediatr Endocrinol [Internet]. 2014;6(3):129-43.

15. World Health Organization. Growth reference data for children aged under 5 years. WHO reference, 2007

16. World Health Organization. Growth reference data for children aged between 5 and 19 years. WHO reference, 2007.

17. Taylor RW, Jones IE, Williams SM, Goulding AG. Evaluation of Waist Circumference, Waist To Hip Ratio, and the Conicity Index As Screening Tools for High Trunk Fat Mass, As Measured By Dual Energy X-Ray Absorptiometry in Children Aged 3-19Y. Am J Clin Nutr. 2000;72(2):490-5.

18. Santomauro F, Lorini C, Pieralli F, Niccolai G, Picciolli P Vezzosi S, et al. Waist-to-height ratio and its associations with body mass index in a sample of Tuscan children in primary school. Italian Journal of Pediatrics. 2017; 43-53.

19. Ashwell M, Hsieh SD. Six reasons why the waist-toheight ratio is a rapid and effective global indicator for health risks of obesity and how its use could simplify the international public health message on obesity. Int $\mathrm{J}$ Food Sci Nutr. 2005;56(5):303-7.

20. De Onis M, Onyango AW, Borghi E, Siyam A, Nishida C, Siekmann J. Development of a WHO growth reference for school-aged children and adolescents. Bull World Health Organ. 2007;85(9):660-7.

21. dos Passos DR, Gigante DP, Maciel FV, Matijasevich A. Comportamento alimentar infantil: comparação entre crianças sem e com excesso de peso em uma escola do município de Pelotas, RS. Rev Paul Pediatr [Internet]. 2015;33(1):42-9. 
22. de Souza WC, de Lima VA, Mascarenhas LPG. Frequência de excesso de peso em pré-escolares. Cinergis. 2016;17(1):44-48.

23. Cunningham SA, Kramer MR, Narayan KMV. Incidence of childhood obesity in the United States. N Engl J Med [Internet]. 2014;370(17):1660-1.

24. Moreira M de A, Cabral PC, Ferreira H da S, de Lira PIC. Excesso de peso e fatores associados em crianças da região nordeste do Brasil. Jornal de Pediatria (Rio de Janeiro). 2012;88(4):347-52.

25. Soares LR, Luiza M, Pereira C, Mota MA, Jacob A, Yuri $\mathrm{V}$, et al. A transição da desnutrição para a obesidade. Braz J Surg Clin Res. 2014;5(1):64-8.

26. da Cunha NS. Alimentação e estado nutricional de préescolares. Universidade Federal do Rio Grande do Sul; 2013.

27. Balduino D. Proposta de ponto de corte para obesidade central, prevalência e correlação da obesidade central e geral em escolares de 6 a 13 anos do município de Xanxerê, SC. 2016
28. lampolsky MN, de Souza FIS, Sarni ROS. Influência do índice de massa corporal e da circunferência abdominal na pressão arterial sistêmica de crianças. Rev Paul Pediatr. 2010;28(2):181-7.

29. Dias LCGD, Cintra RMG de C, Arruda CM, Nunes CM, Gomes CB. Relação entre circunferência abdominal e estado nutricional em pré-escolares de Botucatu, SP. Rev Ciência em Extensão. 2013;9(1):95-104.

\section{Correspondência}

Diego Moura Tanajura Universidade Federal de Sergipe; Campus Prof. Antônio Garcia Filho Av. Governador Marcelo Déda, 13, Centro. Lagarto - Sergipe - Brasil CEP: 49400-000

Email: Diegomouratanajura@gmail.com 